PSICOLOGIA, SAÚDE \& DOENÇAS, 2021, 22(1), 14-24

ISSN - 2182-8407

Sociedade Portuguesa de Psicologia da Saúde - SPPS - www.sp-ps.pt

DOI: http://dx.doi.org/10.15309/21psd220103

PSICOLOGIA POSITIVA 2.0

\author{
José Pais-Ribeiro ${ }^{1,2}$ \\ ${ }^{1}$ WJCR, ISPA - Instituto Universitário. Lisboa, Portugal, jlpr@fpce.up.pt \\ ${ }^{2}$ FPCE-U, Porto, Portugal
}

\begin{abstract}
RESUMO: No virar do século formalizou-se um movimento da Psicologia Positiva (PP) que, sendo também Psicologia e que a acompanhava desde os tempos de William James, não tinha sido assumida com uma amplitude tão geral como aconteceu agora. De facto, a psicologia, respondendo às necessidades da Segunda Grande Guerra, aos tempos de crise que a precederam e aos tempos posteriores de evolução social, tinha dado mais atenção aos aspetos negativos da vida, às falhas que as pessoas tinham, aos seus defeitos. É a partir dos anos revolucionários da década de 60 do século passado que novas formas de pensar a psicologia se estruturaram, nomeadamente passar a olhar para os aspetos positivos, como seja a promoção da saúde ou o bem-estar. Este movimento da Psicologia Positiva teve grande impacto e deu origem a fortes discussões entre os psicólogos. Essas discussões enriqueceram e clarificaram a PP, tornando-a mais abrangente. Hoje, não há área da psicologia que não toque, de algum modo, a PP. É esta história que nos propomos abordar aqui.
\end{abstract}

Palavra-Chave: Psicologia positiva, história da psicologia, bem-estar

\title{
POSITIVE PSYCHOLOGY 2.0
}

ABSTRACT: At the turn of the century, a Positive Psychology (PP) movement was formalized, which, since it was also Psychology and which had followed it since the times of William James, had not been taken on as broadly as it did now. In fact, psychology, responding to the needs of World War II to the times of crisis that preceded it and to the later times of social evolution, had given more attention to the negative aspects of life, to the failures that people had, to their defects. It is from the revolutionary years of the $60 \mathrm{~s}$ of the last century that new ways of thinking about psychology were structured, namely, to start looking at positive aspects, such as health promotion or well-being. This movement of Positive Psychology had a great impact and gave rise to strong discussions among psychologists. These discussions enriched and clarified the PP, making it more comprehensive. Today, there is no area of psychology that does not touch PP in any way. It is this story that we propose to address here.

Keywords: Positive psychology, history of psychology, well-being

Recebido em 15 de setembro de 2020/ Aceite em 03 de fevereiro de 2020

O título deste artigo copia o de Wong (2011), onde o autor propõe uma segunda vaga de psicologia positiva (PP) a que se refere como PP 2.0. A PP, que seria a 1.0 (PP 1.0) nasce formalmente com um texto de Seligman e Csikszentmihalyi (2000), e tem sido seguida por um grande grupo de psicólogos. 


\section{PSICOLOGIA POSITIVA}

Desde o texto formal de lançamento da PP 1.0 ela tem sido discutida, criticada, apoiada, por vários autores e correntes; seja porque ignora a Psicologia Humanística (Waterman, 2013), seja pelos aspetos negativos da PP 1.0 (Held, 2004), seja porque ignora os que abordaram o tema no passado (Cowen \& Kilmer, 2002), seja porque faz uma falsa separação entre positivo e negativo em vez de os integrar (Lazarus, 2003), entre outros. Outros autores (Lomas \& Ivtzan, 2016) falam também em "segunda onda" (second wave PP ou SWPP) na psicologia positiva.

Os autores da PP 1.0 como seja Lee Duckworth et al. (2005) reconheciam os" Distinguished Ancestors and Contemporary Cousins" (pp.632), fazendo referência aos precursores da PP, e o próprio Seligman et al., (2005) refere o trabalho pioneiro desses psicólogos positivos que os antecederam. Na verdade, a PP, seja um movimento, um domínio, um novo paradigma, uma subdisciplina (Marujo et al., 2013), ou simplesmente uma ideologia (Lazarus, 2003), não é nova na nossa cultura nem na psicologia. A ideia que suporta a PP era defendida no início do século XX por William James. Froh (2004) refere que James foi o primeiro psicólogo da PP, e que este argumentava que, a fim de estudar o funcionamento humano ótimo, devia-se considerar a experiência subjetiva da pessoa, evidenciando o seu profundo interesse na subjetividade do indivíduo, e defendendo que a objetividade se baseia numa forte subjetividade. A PP surge depois com Maslow (1954) que, na primeira edição do seu livro "Motivation and Personality ", incluía um último capítulo denominado "Towards a positive psychology". Neste, Maslow afirma que a psicologia em si não foca uma abordagem precisa sobre o potencial humano, e tende a não focar o nível de realização máxima. Dizia nesta primeira edição, que

"The science of psychology has been far more successful on the negative than on the positive side; it has revealed to us much about man's shortcomings, his illnesses, his sins, but little about his potentialities, his virtues, his achievable aspirations, or his full psychological height. It is as if psychology had voluntarily restricted itself to only half its rightful jurisdiction, and that the darker, meaner half" (Maslow, 1954, p. 354).

Nas edições seguintes Maslow retirou este capítulo do livro.

Afirmação idêntica à de Maslow pode ler-se na introdução do texto que lançou oficialmente a PP da autoria de Seligman e Csikszentmihalyi, (2000) afirmação que repete em 2014 (Seligman \& Csikszentmihalyi, 2014). Ainda na década de 50 Marie Jahoda (1958) faz uma abordagem aprofundada ao conceito de "Positive Mental Health", o que é considerado um percursor natural do movimento de psicologia positiva atual (Cowen \& Kilmer, 2002). Já na década de 70 Antonovsky (1979) utiliza o termo "salutogenesis" para contrariar os termos comuns na época de "pathology" e "pathogenesis". Na esfera religiosa Norman Vincent Peale publica em 1996 um livro intitulado "The power of positive thinking" cujos princípios se parecem sobrepor, de algum modo, ao movimento da PP. Seligman (2019) reconhece formalmente parte das falhas que são apontadas ao movimento PP.

Seligman e Csikszentmihalyi (2000) dizem no documento fundador, que a PP se refere ao estudo da experiência subjetiva positiva, dos traços individuais positivos, e que foca o bem-estar social e individual. Lee Duckworth et al. (2005), referem que a PP é o estudo científico das experiências positivas, dos traços individuais positivos, e das instituições que facilitam o seu desenvolvimento. Ou seja, o foco é no positivo parecendo ignorar o negativo.

Na sequência do desenvolvimento da PP Peterson e Seligman (2004) desenvolveram o Manual de Forças e Virtudes de Carácter (MFVC) que, dizem, é um complemento da PP ao Diagnostic and Statistical Manual of Mental Disorders (DSM) e à International Classification of Diseases (ICD) que no capítulo V ou secção F inclui a "Classification of Mental and Behavioural Disorders: Clinical descriptions and diagnostic guidelines", secção que se assemelha ao DSM. Estes manuais propõemse categorizar as perturbações humanas enquanto o MFVC, classifica as forças humanas positivas no seguimento das propostas da PP. Peterson e Seligman (2004) utilizam 10 critérios para, através de discussão (brainstorming) e de revisão de literatura, identificar uma classificação de seis virtudes 
centrais, a saber: Sabedoria e Conhecimento; Coragem; Humanização; Justiça; Sobriedade (temperance); Transcendência. Cada uma inclui subdimensões, no total de 24 forças. A avaliação é feita com um questionário de 240 itens (10 por cada força). Park et al. (2004) explicam que as forças de carácter se podem definir como traços positivos que se refletem nos pensamentos, sentimentos e comportamentos, que existem em graus diferentes e podem ser medidos como diferenças individuais. Mais tarde, Seligman (2011) forneceu uma nova estrutura de bem-estar sugerindo cinco dimensões que abrangem aspetos subjetivos e eudemónicos do bem-estar: Emoções Positivas, Engajamento, Relacionamentos Positivos, Significado, Realização (formando a sigla em inglês PERMA). Estudou a relação entre o MFVC e o PERMA verificando que todas as forças se encontravam positivamente relacionados com todas as dimensões, embora com diferenças de magnitude nessas relações, resultados idênticos encontrados por Wagner et al. (2020).

No entanto se em vez de olharmos os manuais de perturbações ou doenças, como o DSM e o ICD, observarmos as classificações de personalidade, estes assemelham-se mais ao MFVC. Tanto as classificações de personalidade como o MFVC avaliam traços de personalidade, embora o MFVC se foque nos traços positivos. P.ex. um manual clássico, o Revised NEO Personality Inventory iniciado com o estudo "The Baltimore Longitudinal Study of Aging", um estudo longitudinal iniciado em 1958 pelo National Institutes of Health (NIH) nos Estados Unidos da América, em que Paul Costa era um dos investigadores. No âmbito deste estudo Costa e McCrae (1984) investigam a relação entre os determinantes do bem-estar, usando a versão inicial do NEO Personality Inventory que então incluía três fatores (Neuroticismo, Extroversão, Abertura à Experiência) onde confirmavam a relação estatisticamente significativa entre dimensões da personalidade, principalmente o neuroticismo e a extroversão, e as medidas de bem-estar. A versão final da teoria desenvolvida por estes autores inclui cinco fatores ou dimensões da personalidade, Neuroticismo, Extroversão, Abertura à Experiência, Amabilidade, e Conscienciosidade, em que cada dimensão inclui seis facetas, no total de 30 facetas. Destas dimensões o neuroticismo poderia ser considerada a expressão de uma dimensão negativa, mas todas as outras, principalmente a extroversão, exprimem dimensões positivas. Monteiro et al. (2015) mostram correlações negativas entre o neuroticismo e todas as outras quatro dimensões, com magnitude estatisticamente significativa $(p<0,001)$ com a extroversão, a que, dentro destas, poderia ser designada como orientação positiva. DeNeve e Copper (1998) encontraram uma correlação negativa forte entre o Bem Estar Subjetivo e o Neuroticismo. Ou seja, se em vez de se pensar numa classificação de perturbações/ doenças, se pensar numa classificação de funcionamento humano como faz o MFVC ou a personalidade segundo o Revised NEO Personality Inventory, verificamos um padrão semelhante em termos de positividade e negatividade.

\section{A Positividade no virar do século}

No virar do século o termo "positive" passou a ser utilizado em diferentes contextos como, p.ex., "Positive Clinical Psychology" (Wood \& Tarrier, 2010), "Positive Health" (Ryff \& Singer, 1998; Seligman, 2008),'Positive Mental Health" (Jahoda, 1958; Lluch, 2002; Westerhof \& Keyes, 2010), "Positive Epidemiology" (VanderWeele et al., 2020), "Positive Education" (Norrish et al., 2013), "Positive Psychiatry" (Jeste et al., 2015), "Positive Neuroscience" (Greene \& Seligman, 2016), "Positive Organizations" (Cameron \& McNaughton, 2014) "Positive Economics" (Fox, 2012), "Positive Government" (Al Maktoum, 2017), "Positive Theology" (Yaden et al., 2015), entre outros. Ou seja, o virar de século parece consumar uma mudança de paradigma numa grande variedade de domínios salientando uma perspetiva positiva da vida, que recomenda um maior foco nos aspetos positivos em vez dos negativos, ou olhar o copo como meio cheio em vez de meio vazio.

No âmbito da saúde, no virar do século ou mais especificamente em 2001, a Organização Mundial de Saúde aprova, na 54 Assembleia Mundial de Saúde (resolução WHA 54.21) o documento "Classificação Internacional de Funcionalidade, Incapacidade e Saúde" (ICF do acrónimo em inglês 
para International Classification of functioning) que substitui o anterior documento "Classificação Internacional de Deficiências, Incapacidades e Limitações" que tinha sido apresentado em 1976. Nesta alteração de nomenclatura e estrutura, também se propõe alterar a perspetiva da deficiência para a eficiência, ou seja, na avaliação da saúde ou da funcionalidade no campo da saúde, importa salientar o que as pessoas são capazes de fazer e não o que não são capazes de fazer. Esta classificação, diz o manual da OMS, é uma classificação de saúde e de domínios relacionados com a saúde. São "componentes da saúde", dizem, enquanto a classificação da ICD proporciona um "diagnóstico" de doenças, de perturbações ou outras condições. ICF e ICD são complementares explica a OMS. Na continuação da narrativa anterior um exprime uma orientação negativa e a outra uma orientação positiva (OMS/ DGS, 2004).

\section{A PP e a saúde}

Dada a definição formal de saúde da OMS (estado de bem-estar físico, mental e social total e não apenas a ausência de doença ou incapacidade) ainda em vigor hoje, a PP e a saúde devem estar ligadas. De facto, a investigação tem-no mostrado. P.ex., Schmitt e Jüchtern (2001) salientam que o funcionamento psicológico positivo parece ser um dos indicadores mais importantes do bem-estar, o que vai em favor de uma perspetiva da PP. Marques et al, (2011) num estudo longitudinal verificou, dois anos depois, que as variáveis positivas se relacionavam de modo estatisticamente significativo com medidas de saúde mental e com a realização académica. Seligman $\left(2002^{a}\right)$ diz que os objetivos da PP são contribuir para uma mudança na psicologia das preocupações unicamente com a reparação das coisas más da vida, mas para, também, construir a melhor qualidade da vida. A PP tem tido bastante aceitação no seio da psicologia em todo o mundo (Reppold et al., 2019).

No documento fundador da PP Seligman e Csikszentmihalyi (2000) referem que "in the past decade, psychologists have become concerned with prevention" (p.7), e Seligman utiliza exatamente a mesma frase dois anos depois $\left(2002^{\mathrm{a}}\right.$, p.4). Num texto publicado em 2006, Pais Ribeiro comentava que esta afirmação pode parecer estranha para quem tem estado ligado à psicologia da saúde (PS) e reconhece, na definição clássica de PS de Mattarazzo (1980, 1982), que a prevenção das doenças é parte integrante do domínio da PS. De facto, recordando esta definição, a PS é o domínio da psicologia que recorre aos conhecimentos provenientes das diversas áreas da psicologia com vista à promoção e proteção da saúde, à prevenção e tratamento das doenças, à identificação da etiologia e diagnóstico relacionados com a saúde, com as doenças e disfunções associadas, à análise e melhoria do sistema de cuidados de saúde e ao aperfeiçoamento da política de saúde. Portanto não é verdade, como Seligman e Csikszentmihalyi referem que a psicologia se começou a interessar pela prevenção na última década do século XX.

A ideia de promoção da saúde e de prevenção das doenças destaca-se na década de 70 na Declaration of Alma-Ata (1978), uma Conferência Internacional sobre os Cuidados de Saúde Primários, organizada pela Organização Mundial de Saúde, reunião que no século XX se tornou um marco no campo da saúde pública. Este documento, que também é um documento fundador, chama a atenção para a necessidade urgente dos governos, dos profissionais de saúde, e da comunidade em geral, darem importância aos cuidados de saúde primários, nomeadamente à promoção da saúde e à prevenção das doenças. Cerca de dez anos depois a OMS, na sequência da declaração de Alma-Ata, organiza em 1986 na cidade de Ottawa no Canadá, a The first International Conference on Health Promotion, que lança uma serie de ações dirigidas às organizações internacionais, aos governos nacionais e às comunidades locais para alcançar o objetivo da saúde para todos no ano 2000, que tinha sido proposto na conferência de Alma-Ata.

Então, porque é que psicólogos reconhecidos pela sua intervenção na área da saúde, embora na saúde mental, como o é o caso de Seligman, referem que, na década de 90 os psicólogos descobriram 
a prevenção das doenças quando, de facto, esta se tornou, a par da promoção da saúde, um dos fatores associados à emergência da PS 20 anos antes.

\section{A PP como organização}

Os membros da Associação Americana de Psicologia (APA) organizam-se em grupos de interesse, constituindo 54 divisões (ativas). Umas representam subdisciplinas da psicologia (p. ex., experimental, social ou clínica) enquanto outras focam áreas ou tópicos (p. ex., envelhecimento, minorias étnicas, trauma).

Enquanto movimento, a PP não constitui nenhuma das 54 divisões da APA embora seja referida na divisão 17, de consulta psicológica (counseling psychology), onde, informa o seu site:

"In some form Counseling Psychology always has been a vital part of promoting good health and preventing disease, including mental, physical, and social disorders for individuals and communities. It is in the context that this Section was formed. The aim of this group is to focus on how Counseling Psychology fosters and builds human strength and well-being and in pursuing this endeavor, furthers the development of positive psychological science and practice".

Há também um grupo de interesse, "The Positive Psychology Special Interest Group of Division 32" (Society for Humanistic Psychology). Não haver entre as dezenas de divisões da APA uma específica para a PP pode significar uma de duas coisas: ou não está suficientemente madura para dar origem a uma divisão, ou está tão ligada a toda a psicologia, que interessa a todas ou à maioria das divisões.

\section{Discussão sobre a PP}

A discussão sobre a PP tem sido profícua e variada, como seja Cowen e Kilmer (2002), Froh 2004, Held (2004), Lazarus (2003), Wong (2011), entre muitos outros, e algumas com críticas duras como as de Taylor (2001). Esta discussão tem sido empenhada e clarificadora. Por exemplo, Held (2004) apresenta vários tópicos que discute, a saber: "the tyranny of the positive attitude and positive psychology's dominant message", "the negativity of positive psychologists" que subdivide em "Negativity About Negativity" e "Negativity About the Wrong Kind of Positivity: "Unscientific Positivity" and Positive Psychology's "Reality Problems", e "the wisdom of William James".

Uma das discussões organizadas sobre este tema, vem a propósito de um texto de Lazarus, (2003), que deu origem a uma publicação ("Does the Positive Psychology Movement Have Legs?") com a intervenção de alguns dos principais utilizadores e defensores da PP, que nos leva a sugerir que a leiam. É um número da Psychological Inquiry, onde os textos são apresentados. O elemento desencadeador é um texto de Lazarus (2003), acerca do movimento ideológico, como ele o designa, da PP. Lazarus recorda que qualquer emoção pode ter uma valência positiva ou negativa dependendo do contexto em que ela ocorre. Diz que não há nada errado em dar mais atenção ao positivo, desde que não seja à custa do negativo. Uma das críticas que faz à PP é que ela faz uma dicotomia falsa entre positivo e negativo em vez de os integrar. De qualquer modo, refere, emoções positivas podem nem sempre ser positivas e vice-versa. Salienta que o coping joga um papel fundamental na gestão do equilíbrio entre emoções negativas e positivas. O elemento central da posição de Lazarus é a apreciação (appraisal) que cada pessoa faz da situação em que se encontra. Uma visão positiva da vida depende do processo de coping, que pode integrar o bem e o mal, o positivo e o negativo e, muitas vezes, transcender o negativo. Richard Lazarus, entretanto, falecido no início do presente século, é um autor de referência no âmbito do stresse e do coping, com o papel mediador da apreciação (appraisal) primária e secundária que, em conjunto, vão determinar a reação pessoal ao acontecimento stressor que iniciou o processo. 


\section{PSICOLOGIA POSITIVA}

Um texto clássico de Holmes e Rahe (1967), "The Social Readjustment Rating Scale", desenvolveram uma investigação em que examinaram os registos médicos de mais de 5000 doentes, procurando uma associação com acontecimentos de vida stressantes potenciais causadores de doença. Os doentes deviam assinalar numa lista de 43 acontecimentos de vida que tivessem vivenciado. Esses acontecimentos eram pontuados numa escala de 11 a 100, tendo identificado uma associação entre eventos stressantes e doença. Mais tarde Rahe et al., (1970) confirmaram esses dados com outra amostra de 2500 marinheiros. A lista de 43 acontecimentos incluía eventos negativos (p.ex. a morte de um amigo) e positivos (p.ex. ir de férias). Tal parece confirmar a perspetiva de Lazarus que não é o acontecimento em si, mas sim o conjunto, evento stressor, apreciação primária e secundária e coping, que conta para o resultado de um evento, uma emoção, positiva ou negativa.

Wong, (2011) que acrescenta o nome PP 2.0 ao movimento da PP, identifica quatro pilares da boa vida: significado (meaning), virtude (virtue), resiliência (resilience) e bem-estar (well-being). Todos estes pilares, afirma, são formatados pela cultura, assumindo que o bem-estar varia com a cultura. Acrescenta à PP 2.0, concluindo que esta se caracteriza por uma perspetiva, equilibrada, interativa, centrada no significado, e transcultural. Este autor salienta num texto posterior (Wong, 2020) a importância de reconhecer diferentes conceções de bem-estar em diferentes culturas e dependentes desta. P. ex. Stavrova (2019) refere que a investigação aponta para fortes diferenças entre nações nos níveis de bem-estar subjetivo, associados com diferenças entre os povos quanto à prosperidade e outros indicadores socio económicos, políticos e culturais, o que é confirmado por Gardiner et al. (2020) relativamente à felicidade. Schimmack et al. (2002) analisa como a cultura influencia as relações entre emoções positivas (pleasant emotions) e emoções negativas (unpleasant emotions) confirmando a hipótese inicial da investigação que a cultura influenciava a relação entre estas dimensões. Kim-Prieto e Kukoff (2018) verificaram igualmente as diferenças no bem-estar subjetivo em diferentes culturas.

\section{Positivo e Negativo}

Quando falamos em positivo pressupõe-se que existe um negativo, seja na psicologia positiva (versus a negativa), seja no afeto (positivo versus negativo) seja no otimismo (versus pessimismo). No senso comum saúde é o oposto de doença, implicando que quando a pessoa não está doente então está saudável.

Mas a investigação mostra que este modelo simples, de senso comum, não é o mais adequado para compreender a relação entre duas dimensões positivas e negativas. Como já se mostrou antes, a própria OMS apresenta duas classificações, a ICD e a ICF que constituem duas dimensões e não dois polos de uma dimensão. A investigação tem evidenciado também esta bidimensionalidade na saúde. P.ex. a investigação de Keyes (2005; 2007), confirma empiricamente que a saúde mental e a doença mental não constituem polos opostos de um único continuo; antes, eles constituem eixos (dimensões) distintos, e correlacionados $(r=-0,53)$. Explicam que a evidencia aponta para que a ausência de doença mental não implica a presença de saúde mental, e que a ausência de saúde mental não implica a presença de doença mental. Estes dois eixos ou duas dimensões que exprimem, um a saúde mental total versus falta de saúde mental, e outro, doença mental grave versus ausência de doença mental que, na investigação deste autor, partilham 28\% de variância. Keys (2007) designa a saúde mental autêntica como florescimento (flourishing), termo que Fredrickson e Losada (2005) definem como viver numa faixa ótima de funcionamento humano, que conote bondade, generatividade, crescimento e resiliência.

Também Chamberlain (1988) refere haver evidência para a existência de duas dimensões afeto positivo (AP) e afeto negativo (NA). Padrão de bidimensionalidade que também Bradburn (1969), exprime no de bem-estar que, diz é uma função de duas dimensões independentes, AP e NA, que constituem dois fatores correlacionados (Bradburn, 1969; Tellegen et al., 1999; Watson \& Tellegen, 
1985). O AP e o NA constituem variáveis básicas na psicologia. Um texto clássico de Watson e Tellegen, (1985) faz uma revisão de estudos representativos da investigação sobre o afeto que apresentassem os dados de forma suficientemente detalhada para permitir uma reanálise. Esta reanálise mostrou que o NA e o AP, consistentemente, apareciam através de procedimentos complexos, como fatores ou dimensões principais distintas.

Scheier e Carver (1985) desenvolveram um construto "otimismo disposicional", mais o modo de o avaliar, o Life Orientation Test (LOT) e mais tarde a versão reduzida o LOT-R. Na sua investigação assumiram que este construto, o otimismo era unidimensional embora o resultado da sua investigação mostrasse um ajustamento mais adequado com duas dimensões. Dizem que, embora se justifique em muitos casos examinar as duas metades da escala separadamente, afirmam que lhes parece mais razoável tratar as escalas como unidimensionais para a maioria dos propósitos. Carver et al. (2010), a este propósito, referem que tem havido controvérsia se o construto "otimismo disposicional" deverá ser visto como uma dimensão bipolar em que num extremo ficam os otimistas, no meio os neutros e no outro extremo os pessimistas, ou se há duas dimensões separadas, uma referente à dimensão otimismo onde num extremo estão os muito otimistas e no outro extremo ou nada otimistas, a outra dimensão referente ao pessimismo, onde num extremo estão os muito pessimistas e no outro os nada pessimistas. Bailey et al. (2007) encontram duas dimensões, o que tem sido consistente na investigação (Herzberg et al., 2006). Num estudo sobre o otimismo utilizando a versão portuguesa do LOT-R, Pais-Ribeiro et al. (2012), com duas amostras, uma de pessoas com doença crónica (esclerose múltipla) e outra com pessoas da comunidade sem doença, verificaram com recurso à análise fatorial confirmatória, que uma solução unidimensional era mais apropriada para a população com doença crónica, e uma solução bidimensional era mais apropriada para a população da comunidade. Tal como Marshall et al. (1992) e Robinson-Whelen et al. (1997) tinham sugerido, tem havido situações em que separar estes dois atributos levou a uma melhor previsão dos resultados embora refiram que tal não é universal. Mais tarde Carver e Scheier (2014), continuam a salientar esta interpretação dicotómica (unidimensional versus bidimensional), assunto que, afirmam, permanece sem solução, e que é comum a praticamente todas as escalas de traços bipolares, que normalmente formam dois fatores se as escalas contiverem subconjuntos de itens que afirmam ambos os polos. Mas continuam a afirmar a sua unidimensionalidade.

\section{A PP hoje}

Para além da proposta de Wong (2011), para uma PP 2.0, ou Lomas e Ivtzan (2016) que referem uma "segunda onda" (second wave PP ou SWPP) da psicologia positiva, outros têm apontado para perspetivas semelhantes que envolvem uma dinâmica complexa das experiências positivas e negativas. De facto, já na PP 1.0 alguns dos autores principais deste movimento defendiam a integração das experiências negativas e positivas. P.ex. Aspinwall e Staudinger (2003) explicam que a psicologia das forças humanas (human strengths) não é sobre como evitar ou ignorar a experiência negativa, mas sim como a experiência positiva e negativa se relacionam. Dizem estas autores que algumas perspetivas sugerem que o positivo e o negative estão interligadas e interdependentes, e que a experiência humana parece constituir uma dialética básica. Os pais da PP já o expressavam em 2005 "A Intenção é possuir uma maior compreensão e um mais completo balanço científico da experiência humana - os altos e os baixos, e tudo entre eles. Acreditamos que uma ciência completa e uma prática completa da psicologia deverá incluir a compreensão do sofrimento e da felicidade, assim como da sua interação, e intervenções validadas que tanto aliviem o sofrimento como aumentem a felicidade - duas realizações separadas" (Seligman, et al., 2005, p. 410). Seligman (2002b) explicava que a perspetiva da PP significa estar tão focado na força quanto na fraqueza, tão interessado em construir as melhores coisas da vida quanto em reparar o pior, e tão preocupados em cuidar da vida de pessoas normais quanto em curar as feridas dos que necessitam. 


\section{PSICOLOGIA POSITIVA}

Ou seja, parece hoje claro que a PP já não ignora a existência do negativo focando só o positivo como sugeria a definição original de Seligman e Csikszentmihalyi (2000), quando afirmavam que a PP se refere ao estudo da experiência subjetiva positiva, dos traços individuais positivos, e que foca o bem-estar social e individual. Um aspeto que se torna fundamental é que o que torna a experiência positiva é a capacidade de lidar com as situações no sentido em que o coping tem sido abordado, ele próprio multiplexo.

A PP é basicamente psicologia como lembram Soria e Gumbau (2016), ou seja, a PP é hoje uma perspetiva que cobre toda a psicologia, e não só esta como se mostrou antes, e que vem completar o rico campo da psicologia que, de facto, em meados do século passado prestavam demasiada atenção ao lado negativo da vida humana, quase ignorando o lado positivo. Mas a resposta não seria, como parecia ser a definição original de 2000 centrar-se somente no positivo.

\section{REFERÊNCIAS}

Al Maktoum, M. B. R. (2017). Reflections on happiness \& positivity. Explorer Publishing.

Antonovsky, A. (1979). Health, stress and coping. Jossey-Bass

Aspinwall, L. G., \& Staudinger, U. M. (2003). A psychology of human strengths: Some central issues of an emerging field. In L. G. Aspinwall \& U. M. Staudinger (Eds.), A psychology of human strengths: Fundamental questions and future directions for a positive psychology (pp. 9-22). American Psychological Association.

Bailey, T. C., Eng, W., Frisch, M. B., \& Snyder, C. R. (2007). Hope and optimism as related to life satisfaction. The Journal of Positive Psychology, 2(3), 168-175. https://doi.org/10.1080/17439760701409546

Bradburn, M. (1969). The Structure of Psychological Well-Being. Aldine Pub. Co.

Cameron, K., \& McNaughton, J. (2014). Positive organizational change. Journal of Applied Behavioral Science, 50, 445-462 https://doi.org/10.1177/0021886314549922.

Carver, C. S., \& Scheier, M. F. (2014). Dispositional optimism. Trends in cognitive sciences, 18(6), 293-299. https://doi.10.1016/j.tics.2014.02.003

Carver, C. S., Scheier, M. F., \& Segerstrom, S. C. (2010). Optimism. Clinical psychology review, 30(7), 879-889. http://dx.doi.org/10.1016/j.cpr.2010.01.006

Chamberlain, K. (1988). On the structure of subjective well-being. Social indicators research, 20(6), 581-604. https://doi.org/10.1007/BF03359559

Costa, P. T., \& McCrae, R. R. (1984). Personality as a Lifelong Determinant of Well-Being. In C. Malatesta, \& C. Izard (Eds.), Affective Process in Adult Development and Aging (pp. 141-157). Sage.

Cowen, E. L., \& Kilmer, R. P. (2002). "Positive psychology": Some plusses and some open issues. Journal of community psychology, 30(4), 449-460. https://doi.10.1002/jcop.10014

Declaration of Alma-Ata. (1978). International conference on primary health care. WHO Chron, $32(11), 428-430$ ?

DeNeve, K., \& Copper, H. (1998). The Happy Personality: A Meta-Analysis of 137 Personality Traits and Subjective Well-Being. Psychological Bulletin, 124(2), 197-229. https://doi.org/10.1037/0033-2909.124.2.197

Fox, J. (2012). The economics of well-being. Harvard Business Review, 90(1-2), 78-83.

Fredrickson, B. L, \& Losada, M. F. (2005). Positive affect and complex dynamics of human flourishing. American Psychologist. 60 (7), 678-686. https://doi.10.1037/0003-066x.60.7.678

Froh, J. J. (2004). The history of positive psychology: Truth be told. NYS psychologist, 16(3), 18-20. 
Gardiner, G., Lee, D., Baranski, E., Funder, D., \& Members of the International Situations Project (2020). Happiness around the world: A combined etic-emic approach across 63 countries. PLoSONE15(12): e0242718. https://doi.org/10.1371/journal.pone.0242718

Greene, J. D., \& Seligman, M. E. (Eds.). (2016). Positive neuroscience. University Press.

Held, B. S. (2004). The negative side of positive psychology. Journal of humanistic psychology, 44(1), 9-46. https://doi.org/10.1177/0022167803259645

Herzberg, P. Y., Glaesmer, H., \& Hoyer, J. (2006). Separating optimism and pessimism: A robust psychometric analysis of the Revised Life Orientation Test (LOT-R). Psychological assessment, 18(4), 433-438. https://doi.org/10.1037/1040-3590.18.4.433

Holmes, T. H., \& Rahe, R. H. (1967). The Social Readjustment Rating Scale. Journal of Psychosomatic Research, 11(2), 213-218. https://doi.org/10.1016/0022-3999(67)90010-4

Jahoda, M. (1958). Current concepts of positive mental health. Basic Books

Jeste, D. V., Palmer, B. W., Rettew, D. C., \& Boardman, S. (2015). Positive psychiatry: Its time has come. The Journal of Clinical Psychiatry, 76(6), 675-683. https://doi.10.4088/JCP.14nr09599

Keyes, C. L. (2005). Mental illness and/or mental health? Investigating axioms of the complete state model of health. Journal of consulting and clinical psychology, 73(3), 539-548. https://doi.org/10.1037/0022-006X.73.3.539

Keyes, C. L. (2007). Promoting and protecting mental health as flourishing: A complementary strategy for improving national mental health. American psychologist, 62(2), 95-108. https://doi.org/10.1037/0003-066X.62.2.95

Kim-Prieto, C., \& Kukoff, J. (2018). Subjective well-being across cultures: Some universals and many more differences. In K. D. Keith (Ed.), Culture and psychology. Culture across the curriculum: A psychology teacher's handbook (p. 464-477). Cambridge University Press. https://doi.org/10.1017/9781316996706.025

Lazarus, R. S. (2003). Does the positive psychology movement have legs? Psychological inquiry, 14(2), 93-109.https://doi.org/10.1207/S15327965PLI1402_02

Lee Duckworth, A., Steen, T. A., \& Seligman, M. E. (2005). Positive psychology in clinical practice. Annual Review of Clinical Psychology., 1, 629-651. https://doi.org/10.1146/annurev.clinpsy.1.102803.144154

Lluch, M. T. (2002). Evaluación empírica de un modelo conceptual de salud mental positiva. Salud Mental, 25(4), 42-55.

Lomas, T., \& Ivtzan, I. (2016). Second wave positive psychology: Exploring the positive-negative dialectics of wellbeing. Journal of Happiness Studies, 17(4), 1753-1768. https://doi.org/10.1007/s10902-015-9668-y

Marques, S. C., Pais-Ribeiro, J. L. \& Lopez, S. J. (2011). The role of positive psychology constructs in predicting mental health and academic achievement in Portuguese children and adolescents: A 2-year longitudinal study. Journal of Happiness Studies, 12, 1049-1062. https://doi.org/10.1007/s10902-010-9244-4

Marshall, G.N., Wortman, C.B., Kusulas, J.W., Hervig, L.K., \& Vickers, R.R. Jr. (1992). Distinguishing optimism from pessimism: Relations to fundamental dimensions of mood and personality. Journal of Personality and Social Psychology, 62, 1067-1074. https://doi.org/10.1037/0022-3514.62.6.1067

Marujo, H., Neto, L. M., \& de Falé Balancho, L. S. (2013). Emergência, desenvolvimento e desafios da psicologia positiva: Da experiência subjetiva à mudança social. ECOS-Estudos Contemporâneos da Subjetividade, 3(2), 179-201.

Maslow, A. H. (1954). Motivation and personality. Harper \& Row, Publishers.

Matarazzo, J.D. (1980). Behavioral health and behavioral medicine. American Psychologist, 35(9),807-817. http://dx.doi.10.1037//0003-066x.35.9.807. 
Matarazzo, J.D. (1982). Behavioral health's challenge to academic, scientific and professional psychology. American Psychologist, 37(1), 1-14. https://doi.org/10.1037/0003-066X.37.1.1

Monteiro, S., Almeida, L. D., Cruz, J., \& Franco, A. (2015). The relationship of personality, study practice and learning environments on excelent engineering students. Análise Psicológica, 33(1), 97-111. http://dx.doi.org/10.14417/ap.953

Norrish, J. M., Williams, P., O’Connor, M., \& Robinson, J. (2013). An applied framework for positive education. International Journal of Wellbeing, 3(2), 147-161. http://dx.doi.10.5502/ijw.v3i2.2

OMS/ DGS (2004). Classificação Internacional de Funcionalidade, Incapacidade e Saúde (CIF). DGS

Pais-Ribeiro, J. (2006). Relação entre a psicologia positiva e as suas variáveis protectoras, e a qualidade de vida e bem-estar como variáveis de resultado. In: I. Leal (Coord.). Perspectivas em psicologia da saúde (pp.231-244). Quarteto editora

Pais-Ribeiro, J., Pedro, L., \& Marques, S. (2012). Dispositional optimism is unidimensional or bidimensional: study with a Portuguese version of the revised life orientation test. The Spanish Journal of Psychology, 15, 1259-1271. http://dx.doi.org/10.5209/rev_SJOP.2012.v15.n3.39412.

Park, N., Peterson, C., \& Seligman, M. E. (2004). Strengths of character and well-being. Journal of social and Clinical Psychology, 23(5), 603-619. https://doi.org/10.1521/jscp.23.5.603.50748

Peale, N. V. (1996). The power of positive thinking. New York. Ballantine Books

Peterson e Seligman (2004)

Peterson, C., \& Seligman, E. (2004). Character strengths and virtues: a handbook and classification. APA Press.

Rahe, R.H., Mahan, J.L., \& Arthur, R.J. (1970). Prediction of near-future health change from subjects' preceding life changes. Journal of Psychosomatic Research, 14 (4), 401-406. https://doi.10.1016/0022-3999(70)90008-5.

Reppold, C. T., D'Azevedo, L. S., Tocchetto, B. S., Diaz, G. B., Kato, S. K., \& Hutz, C. S. (2019). Avanços da psicologia positiva no Brasil. Psicologia para América Latina, 32, 133-141.

Robinson-Whelen, S., Kim, C., MacCallum, R.C., \& Kiecolt-Glaser, J.K. (1997). Distinguishing optimism from pessimism in older adults: Is it more important to be optimistic or not to be pessimistic? Journal of Personality and Social Psychology, 73,1345-1353. https://doi.org/10.1037/0022-3514.73.6.1345

Ryff, C. D., \& Singer, B. (1998). The contours of positive human health. Psychological inquiry, 9(1), 1-28. https://doi.org/10.1207/s15327965pli0901_1

Scheier, M. F., \& Carver, C. S. (1985). Optimism, coping, and health: assessment and implications of generalized outcome expectancies. Health psychology, 4(3), 219-247. https://doi.org/10.1037/0278-6133.4.3.219

Schimmack, U., Oishi, S., \& Diener, E. (2002). Cultural influences on the relation between pleasant emotions and unpleasant emotions: Asian dialectic philosophies or individualism-collectivism? Cognition \& Emotion, 16(6), 705-719. https://doi.org/10.1080/02699930143000590

Schmitt, M., \& Jüchtern, J. C. (2001). The structure of subjective well-being in middle adulthood. Aging \& mental health, 5(1), 47-55. https://doi.org/10.1080/13607860020020645

Seligman, M. E. P. (2002a). Positive psychology, positive prevention, and positive therapy. In C. R. Snyder \& S. J. Lopez (Eds.), Handbook of positive psychology (p. 3-9). Oxford University Press.

Seligman, M. E. (2002b). Authentic happiness. Free Press

Seligman, M. E. (2008). Positive health. Applied psychology, 57, 3-18. https://doi.org/10.1111/j.1464-0597.2008.00351.x

Seligman, M. E. (2011). Flourish. Free Press

Seligman, M. E. (2019). Positive psychology: A personal history. Annual review of clinical psychology, 15, 1-23. https://doi.10.1146/annurev-clinpsy-050718-095653. 
Seligman, M. E. P., \& Csikszentmihalyi, M. (2000). Positive psychology: An introduction. American Psychologist, 55(1), 5-14. https://doi.10.1037/0003-066X.55.1.5

Seligman, M. E., \& Csikszentmihalyi, M. (2014). Positive psychology: An introduction. In: Flow and the foundations of positive psychology (pp. 279-298). Springer, Dordrecht.

Seligman, M. E., Steen, T. A., Park, N., \& Peterson, C. (2005). Positive psychology progress: empirical validation of interventions. American psychologist, 60(5), 410-421. https://doi.10.1037/0003-066X.60.5.410

Soria, M. S., \& Gumbau, S. L. (2016). Hacia una psicología positiva aplicada. Papeles del psicólogo, $37(3), 161-164$.

Stavrova, O. (2019). How much do sources of happiness vary across countries? A review of the empirical literature. KZfSS Kölner Zeitschrift für Soziologie und Sozialpsychologie, 71(1), 429464. https://doi.org/10.1007/s11577-019-00612-y

Taylor, E. (2001). Positive psychology and humanistic psychology: A reply to Seligman. Journal of Humanistic Psychology, 41(1), 13-29. https://doi.org/10.1177/0022167801411003

VanderWeele, T. J., Chen, Y., Long, K., Kim, E. S., Trudel-Fitzgerald, C., \& Kubzansky, L. D. (2020). Positive epidemiology? Epidemiology, 31(2), 189-193. https://doi. 10.1097/EDE.0000000000001147

Wagner, L., Gander, F., Proyer, R. T., \& Ruch, W. (2020). Character strengths and PERMA: Investigating the relationships of character strengths with a multidimensional framework of wellbeing. Applied Research in Quality of Life, 15(2), 307-328. https://doi.org/10.1007/s11482-0189695-z

Waterman, A. S. (2013). The humanistic psychology-positive psychology divide: Contrasts in philosophical foundations. American Psychologist, 68(3), 124. https://doi.10.1037/a0032168

Watson, D., \& Tellegen, A. (1985). Toward a consensual structure of mood. Psychological Bulletin, 98, 219-235. https://doi.org/10.1037/0033-2909.98.2.219

Westerhof, G. J., \& Keyes, C. L. (2010). Mental illness and mental health: The two continua model across the lifespan. Journal of adult development, 17(2), 110-119. https://doi.10.1007/s10804-0099082-y

Wong, P. T. P. (2011). Positive psychology 2.0: Towards a balanced interactive model of the good life. Canadian Psychology, 52(2), 69-81. https://doi.org/10.1037/a0022511

Wong, P. T. P. (2020). The maturing of positive psychology and the emerging PP 2.0: A book review of Positive Psychology (3rd ed.) by William Compton and Edward Hoffman. International Journal of Wellbeing, 10(1), 107-117. https://doi.10.5502/ijw.v10i1.885

Wood, A. M., \& Tarrier, N. (2010). Positive clinical psychology: A new vision and strategy for integrated research and practice. Clinical psychology review, 30(7), 819-829. https://doi.10.1016/j.cpr.2010.06.003

Yaden, D. B., McCall, T. D., \& Ellens, J. H. (Eds.). (2015). Being Called: Scientific, Secular, and Sacred Perspectives: Scientific, Secular, and Sacred Perspectives. ABC-CLIO. 